\title{
CORRIGENDUM
}

\section{A dose-response relation between aerobic exercise and visceral fat reduction: systematic review of clinical trials}

\author{
K Ohkawara, S Tanaka, M Miyachi, K Ishikawa-Takata and I Tabata
}

International Journal of Obesity (2008) 32, 395; doi:10.1038/sj.ijo.0803749

Correction to: International Journal of Obesity advanced online publication 17 July 2007; doi: 10.1038/sj.ijo.0803683

After the publication of the above paper, the authors have noticed an error in Figure 3, as the position of one plot should be changed and two plots should be erased, while statistical results remain unchanged. The correct figure is shown below.

The authors apologize for the errors.
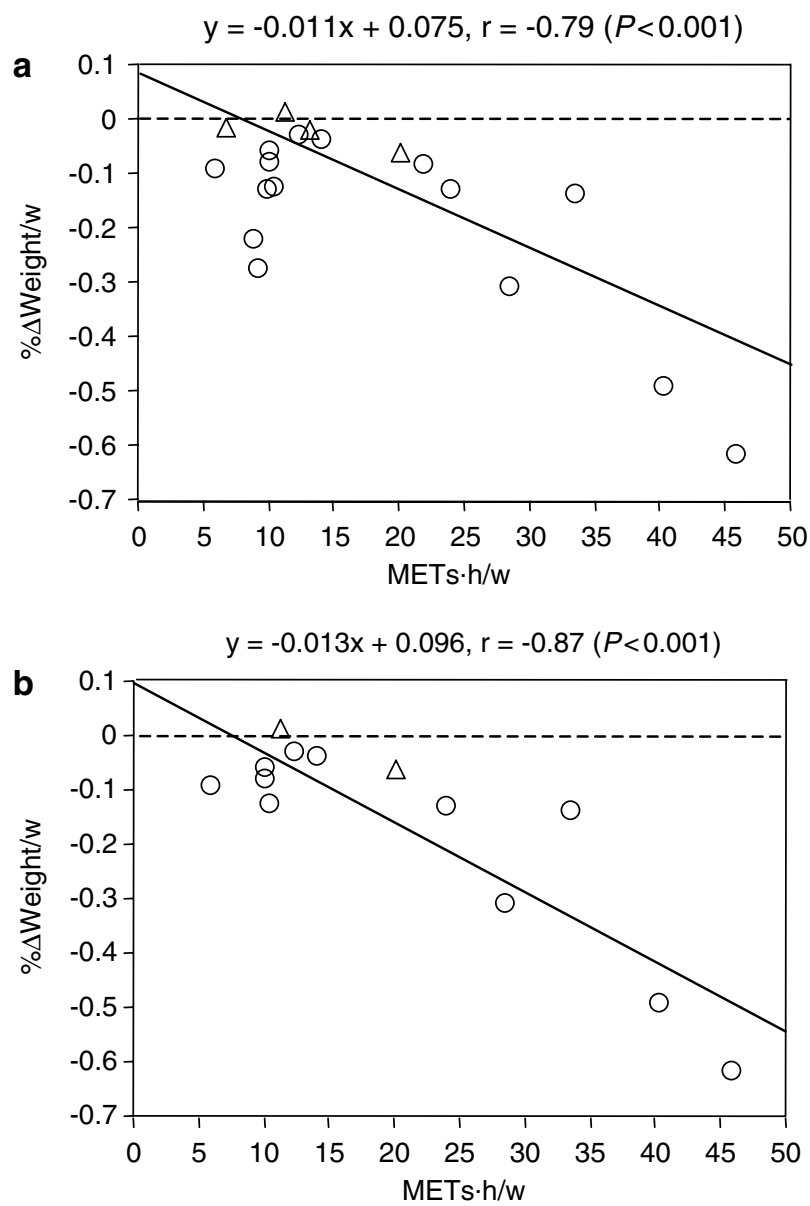

Figure 3 Relations between METs.h/w and \% $\%$ Weight/w during interventions in the all selected groups (a) and after excluding the groups with metabolic-related disorder subjects (b). Abbreviations: METs.h/w, $\Sigma$ (metabolic equivalents $\times$ hour) per week; $\% \Delta$ Weight $/ w$, percentage of weight change per week; $r$, Pearson's correlate coefficient; $O$, the group with a significant visceral fat reduction $(P<0.05) ; \triangle$, the group without a significant visceral fat reduction $(P<0.05)$. The groups without a weight loss intentionally were excluded for these analysis. 